\title{
Handling Concept Drift for Predictions in Business Process Mining
}

\author{
Lucas Baier \\ Josua Reimold \\ Niklas Kühl \\ Karlsruhe Institute of Technology (KIT) Karlsruhe Institute of Technology (KIT) Karlsruhe Institute of Technology (KIT) \\ Karlsruhe, Germany \\ Email: lucas.baier@kit.edu \\ Karlsruhe, Germany \\ Karlsruhe, Germany \\ Email: josua.reimold@alumni.kit.edu \\ Email: niklas.kuehl@kit.edu
}

\begin{abstract}
Predictive services nowadays play an important role across all business sectors. However, deployed machine learning models are challenged by changing data streams over time which is described as concept drift. Prediction quality of models can be largely influenced by this phenomenon. Therefore, concept drift is usually handled by retraining of the model. However, current research lacks a recommendation which data should be selected for the retraining of the machine learning model. Therefore, we systematically analyze different data selection strategies in this work. Subsequently, we instantiate our findings on a use case in process mining which is strongly affected by concept drift. We can show that we can improve accuracy from 0.5400 to 0.7010 with concept drift handling. Furthermore, we depict the effects of the different data selection strategies.
\end{abstract}

\section{INTRODUCTION}

Machine learning plays a major role in the recent developments of artificial intelligence [1]. It is widely considered to be one of the most disruptive technologies in the last decades. Its fast progress is fueled by both the development of new learning algorithms and the huge availability of low-cost computation and data [2]. Machine learning is applied across all sectors and in all functional business areas, such as research and development, marketing or finance [3]. Many companies rely on machine learning models for offering new services or for improving their existing ones [4]. As Davenport [5] has shown, companies leveraging their data sources achieve a substantial competitive advantage. Especially in the area of services, there seems to be large untapped potential in both, research and practice [6], [7].

To address this promising gap, predictive services offer the possibilities to implement machine learning into different application fields [8]. Typically, techniques of supervised machine learning provide the basis for such predictive services [2] which are trained by using historical data of input features and a label. Subsequently, the model is used to continuously compute predictions on a stream of incoming data. However, data streams typically change over time. This is one of the major challenges for applying machine learning in practice [9] since the prediction quality is very sensitive to the input data [10]. Therefore, the problem of changing data stream over time has been examined under the term "concept drift" [11].

Usual strategies for handling concept drift rely on dedicated drift detection algorithms [12]. As soon as a drift is detected, the corresponding machine learning model will be retrained. However, it remains an open research question which data instances for the retraining of the machine learning model should be applied (e.g. data before or after the detection). Therefore, we aim at systematically examining the difference between different retraining options which is expressed in RQ1.

RQ1. Which data should be used for the retraining of a machine learning model when a concept drift is detected?

Subsequently, we apply our findings of RQ1 in a real-life use case in business process mining, a typical example of a predictive service. Business process management in general, and business process mining in particular, have received a lot of attention recently in top management because it improves decision making in organizations [13], [14]. New applications are extended by the use of predictive analytics [15]. Since business processes are inherently dynamic, those new features are largely exposed to concept drift [16]. This requires the adaptation of existing methods to ensure their validity over time. Therefore, we want to examine the effects of the different data options on this use case which is regularly confronted with concept drift in the second research question.

RQ2. What are the effects of the different retraining options in a real-life use case in business process mining?

The remainder of the paper is structured as follows: $\mathrm{Sec}-$ tion II presents related work on which we base our research. Section III introduces different aspects which can be considered for the retraining of a machine learning model after detection of a drift. Section IV] presents the chosen use case as well as the evaluation of the different options discussed in the previous section. The final section discusses our results, describes theoretical and managerial implications, acknowledges limitations and outlines future research.

\section{RELATED WORK}

This section gives a brief overview of related work about concept drift as well as its detection. Furthermore, related work regarding process mining is introduced.

\section{A. Concept Drift}

Machine learning can create ongoing value when the corresponding prediction models are deployed in connected information systems and deliver ongoing recommendations on continuous data streams. However, data streams usually change 
and evolve over time. This is also reflected in changes in the underlying probability distribution or their data structures [17]. The challenge of changing data streams for machine learning tasks has been described with the term "concept drift" [11] in computer science. A concept $p(X, y)$ is defined as the joint probability distribution of a set of input features $X$ and the corresponding label $y[12]$. In real applications, concepts often change with time [10]. This change can be expressed in a mathematical definition as follows [12]:

$$
\exists X: p_{t 0}(X, y) \neq p_{t 1}(X, y)
$$

This definition explains concept drift as the change in the joint probability distribution between two time points $t_{0}$ and $t_{1}$. Therefore, machine learning models built on previous data (in $t_{0}$ ) might not be suitable for making predictions on new incoming data (in $t_{1}$ ). This change requires the frequent adaptation of the prediction approach.

Changes in the incoming data stream can depend on a multitude of different internal or external influences. Usually, it is impossible to measure all of those possible confounding factors in an environment-which is why this information cannot be included in the predictive features of a ML model. Those factors are often considered as "hidden context" of a predictive model [11]. Concept drift is usually classified into the following categories [18]: Abrupt or sudden concept drift where data structures change very quickly (e.g. sensor failure), gradual and incremental concept drift (e.g. change in customers' buying preferences) or seasonal and reoccurring drifts (e.g. A/C sales in summer). There exists also a more finegrained taxonomy [19] which also considers the magnitude of the drift for instance.

A wide variety of approaches for the handling of concept drifts has been proposed [12]. However, most approaches rely on an explicit drift detection which detects changes in the data distribution and triggers corresponding adaptations. Two of the most popular algorithms are Page-Hinkley [20] and ADWIN [21]. Page-Hinkley works by continuously monitoring an input variable (e.g. the input data or the prediction accuracy). As soon as the variable differs significantly from its historical average, a change is flagged. ADWIN, in contrast, is a change detector which relies on two detection windows. As soon as the means of those two windows are distinct enough, a change alert is triggered, and the older window is dropped.

\section{B. Process Mining}

Business process mining is a research discipline that originates from business process modeling and analysis on the one side and data mining on the other side [22]. The goal of process mining is to discover, monitor and improve operational processes by extracting data from event logs [23]. This way, business processes are analyzed in the way as they are really executed [24]. These event logs can be created by extracting the digital traces of business processes that are stored in today's information systems, e.g. ERP or CRM systems [25]. The minimum information needed for an event log is therefore a unique CaseID to identify and differentiate each case and an event with relating timestamp to define the activity of the process. This combination is important, so that the real sequence of the events can be ensured.

Process mining can be differentiated into three types [16] where the first type is discovery. After extraction of the event logs, a process model can be built. This also allows to understand different variants of business processes [26]. The second type is conformance. In this case, existing process models can be compared with an event log of the same process and discrepancies between both can be discovered. The third type relates to enhancement where existing process models are extended. This can also refer to operational support where predictions and recommendations based on prediction models from historic information can be used to optimize running cases [27]. An application could be the prediction of the remaining time of a case [28] or the prediction of the next executed activity in a case [29].Furthermore, there are approaches predicting whether a case will be completed [30]. With such predictions, the organizational procedures can be optimized, and personnel planning is more accurate. For instance, it can be very valuable for a customer to know the remaining process time of his insurance claim or when his product order will arrive.

A very important challenge in process mining is the occurrence of concept drift [16] which refers to processes that are changing while being analyzed. For instance, the sequence of events can change, e.g. two events that occurred in parallel are now occurring one after another. Processes may change due to a variety of reasons, from seasonal effects over market changes to organizational adjustments. Business processes are inherently dynamic over time and therefore prone to change. Nevertheless, concept drift research in business process mining is rather scarce. Sudden concept drift in process mining, such as rearranging or replacing activities, has been examined [22]. The authors propose to detect those drifts by computing correlation between event classes. Another approach proposes a framework which computes dedicated features on the event logs and subsequently compares those features over different windows to detect concept drift [31]. In this context, this method to detect drifts is similar to traditional concept drift approaches described in Subsection II-A More advanced options use an adaptive approach based on a Chi-square test which also allows to detect different types of process drift [32]. Other research aims at better understanding the type or the degree of change [33] or providing more robustness to process drift detection methods [34].

The approaches described above focus on concept drift in the type of event or their order in a process. This is related to the first type of process mining (discovery) that focuses on deriving a process model. The ultimate objective of this analysis is to identify and better understand the activities that trigger process drift in the first place.

However, this analysis does not contain any predictive component. Existing work has not yet considered concept drift in the enhancement type of process mining where predictions based on machine learning are computed to optimize oper- 


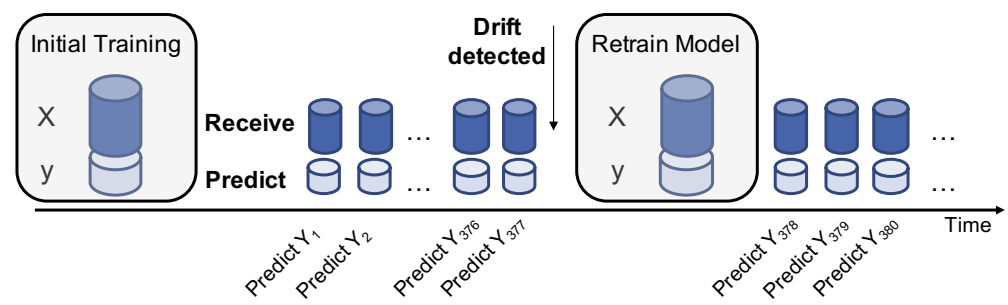

Fig. 1: Depiction of learning mode retraining

ations [27]. Compared to previous work, this also requires strategies for an adaption of prediction models over time.

\section{DATA SELECTION FOR RETRAINING}

This section introduces the two different learning modes for machine learning models and provides an overview on which data can be used for the retraining of a model if the training process has to be started from the beginning.

\section{A. Learning mode}

In the context of data streams and ongoing predictions, two learning modes for machine learning models can be differentiated: retraining and incremental learning [8].

The method of retraining is illustrated in Figure 1. The figure shows that in the beginning the model is trained on an initial batch of data. After the initial model has been trained, new incoming data instances $X$ result in predictions $y$ (e.g., $y_{1}$ in Figure 1). This happens iteratively for every new data instance in the data stream until the drift detection method issues an alert which requires an adaptation of the prediction model. Correspondingly, the old model is discarded, and a completely new prediction model is trained which is subsequently applied to every incoming data instance (e.g., the new prediction model after retraining is applied for the first time by predicting $y_{378}$ and the following data instances in Figure 1 ).

Incremental learning, in contrast, works by continuously updating the prediction model. Comparably, the starting model is trained on an initial data set. When new data instances arrive, the model issues a prediction. However, as soon as the true target label of this data instance is known, this information is used to incrementally improve the prediction model. The main advantage of this approach is that every new labeled instance arriving will be used for model improvement and thus, the model automatically adapts to changing concepts. This approach is comparable to a sliding window approach. In general, the incremental updates will not be computed after a single new data instance has arrived but rather after the reception of a small batch of data instances (e.g. 10 or 20). This reduces the computational complexity. Unfortunately, only few machine learning algorithms such as Naïve Bayes, Neural Networks or Hoeffding Trees [35], [36] implement the opportunity for incremental updates.

Despite the continuous updates of the prediction model, this approach might be confronted with degrading performance over time. For instance, the incremental updates of the model cannot adapt to very quick changes which occur during sudden concept drifts. In this case, it might be also necessary to discard the current model and train a new model. This would depict a combination of both learning modes retraining and incremental updates.

\section{B. Data Selection for Retraining of the Machine Learning Model}

In case of concept drift, the previous model will be discarded, and a new model is trained as depicted in Figure 1 However, when implementing this approach, we need to select the data that is used for the retraining of the machine learning model. So far, literature does not provide any knowledge on which data of the data stream should be used for the retraining of the prediction model. Therefore, we implement and evaluate three different data selection strategies which we call next, mixed and last. The difference between these approaches is depicted in Figure 2.

The approach next is displayed in the upper part of Figure 2 As soon as a concept drift is detected, the model collects the next batch of instances with corresponding labels (e.g. two new data instances in the figure). When this next batch is complete, the retraining is started and subsequently the new model is applied. This also means that the previous model is used to predict the next batch after the concept drift since it is also necessary to issue predictions for those instances (and the new model has not been learned yet). The intuition guiding this approach is that data following a concept drift, complies with the new concept and is therefore an optimal basis for a new model.

The other approaches mixed and last are also displayed in Figure 2. In case of the mixed approach, the model retraining relies on data from before and also after the detection. Compared to the first approach (next), the new model can be applied faster since it requires less data after the concept drift. The last approach entirely relies on data which was acquired before the concept drift detection alert. This means that the new prediction model will be applied right on the next data instances after the detection of a drift. This approach might work well because drift detection algorithms usually work with a slight delay. Therefore, the data batch before the alert might already belong to the new concept.

During the application of our use case, we aim to systematically test all three approaches in order to quantify the 


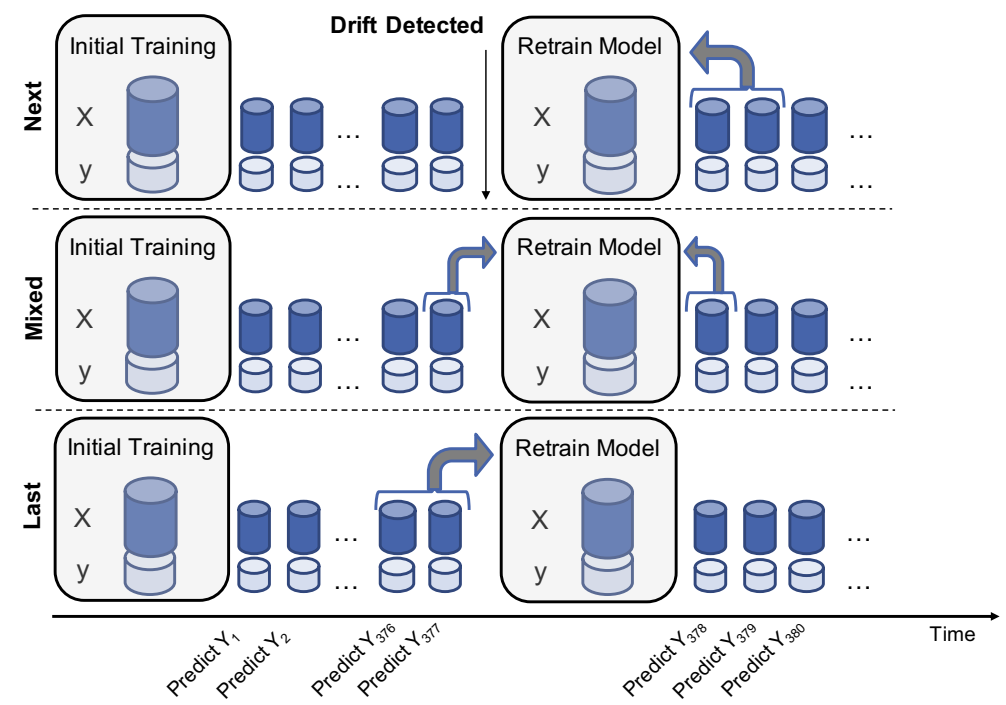

Fig. 2: Three different approaches for retraining of model

differences between those and also to give recommendations for future implementations.

\section{Use Case in Process Mining}

A process mining solution provider gives us access to a data set of the purchase to pay (P2P) service process of a large German company. This process contains all activities related to the procurement of a product or service. A simplified $\mathrm{P} 2 \mathrm{P}$ process starts with the creation of a purchase order and is followed by the reception of the respective goods by the logistics department and the invoice which is then processed over various financial departments in the company. An exemplary process of this $\mathrm{P} 2 \mathrm{P}$ process can be seen in Figure 3.

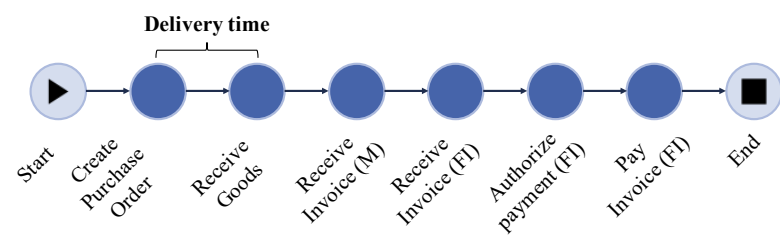

Fig. 3: Typical process variant for a $\mathrm{P} 2 \mathrm{P}$ process

In this use case, we want to predict the throughput time or delivery time (marked in bold) between the creation time of the purchase order and the reception of the goods. This information is quite important for the company since all subsequent process steps such as production can be optimized, and significant cost savings can be realized. The data is extracted from the business intelligence platform Qlik and then preprocessed in Python. The foundation of the data set is an event $\log$ that is enriched with numerous additional attributes to fully describe the process. The attributes are anonymized and transformed to ensure that the data is not retraceable.
In total, we receive data about 70,774 purchase transactions from 2016 until 2018 which we can use to train and evaluate the machine learning approach. Importantly, those transactions are displayed in chronological order, which is a necessary prerequisite for an analysis of concept drift over time.

We use the package scikit-mulitflow [37] as the basis of our analysis since it extends the machine learning package scikitlearn with a stream data framework. It allows to process data sets and simulate them as a data stream. Furthermore, different concept drift detectors are implemented and can be evaluated. We extend the package by implementing the different training modes (last, mixed, next) which we discussed in Section III

\section{A. Data Analysis}

We first perform an exploratory data analysis to analyze the available features and build a predictive model that can be used for the analysis of concept drift in process mining. Table I gives an overview on available features of the data set. Categorical features are one-hot-encoded for the subsequent data processing. Material class refers to the product category of the purchased product. Regarding this feature, we only use the first four numbers of the material class in order to reduce the number of different categories resulting in 123 different categories in total. Furthermore, we have information about the purchase order value. The purchase order value is an important feature for our endeavor since it is a clear indicator of the relevance of the respective purchase order for the company. However, the distribution of the order value is highly skewed which might pose a problem for the prediction model. Therefore, the values are transformed with a Box-Cox transformation [38] into a gaussian distribution.

Other features included in the data set are the country of the bank were the payment is executed and the document type of the purchase order. The document type includes information about different ways to create a purchase order: e.g., the 
TABLE I: Overview of predictive features

\begin{tabular}{lll}
\hline Feature & Type & $\begin{array}{l}\text { Number of items / } \\
\text { Range of values }\end{array}$ \\
\hline Material class & categorical & 123 \\
Document type & categorical & 7 \\
Plant code & categorical & 4 \\
Purchase order value & numerical & $1-458,079$ \\
Supplier & categorical & 799 \\
Bank country & categorical & 18 \\
Supplier country & categorical & 14 \\
Purchasing group & categorical & 75 \\
\hline Throughput time $[\mathrm{h}]$ (Target) & numerical & $1-120,000$ \\
\hline
\end{tabular}

order is created manually by an employee in the purchasing department or is based on existing long-time contracts. Other options include the automatic creation by an MRP-system. The country of the supplier is also relevant for the analysis. Obviously, a purchasing process requires more time if the supplier is located in another country because this leads to additional steps during the sales process such as customs papers, currency conversion or additional insurance of the transport. The feature plant code stores information about the plant which initiated the purchase process. Purchasing group is the department or group at which the purchase order is created and processed. Furthermore, we also have information about the supplier itself who is distributing the requested product.

The target variable in this use case is the throughput time or delivery time of a purchase order. This refers to the amount of time between the first two steps depicted in Figure 3 By considering the delivery time, we ensure that the start of the purchase process is considered as well as the most important event for production and workforce scheduling, namely the arrival of the ordered goods. The prediction of the estimated arrival time of a product is important because planning processes can be optimized with this information. This might result in significant cost savings as well as the minimization of production time due to the optimization of waiting time.

A histogram of the throughput time can be seen in Figure 4 For approximately $50 \%$ of all purchase orders, respective products and goods are received within 14 days $(<336 \mathrm{~h})$. Regarding the remaining purchase orders, another $25 \%$ of those have a delivery time within 60 days. The other purchase orders even have a larger delivery time, up to 537 days.

Due to the challenging distribution of the target variable, we transform the use case into a multi-class classification problem. Although this leads to an abstraction and loss of information, this step is meaningful for an initial analysis of the use case. To transform the target variable, all purchase orders are divided into three equally sized classes of throughput times as can be seen in Table II Therefore, the first class contains purchase orders with a delivery time of up to 6 days. The second class contains purchase orders with a delivery time between 7 and 39 days and the last class contains all cases for which the delivery takes more than 40 days. We train a machine learning model which predicts whether a purchase order will belong to

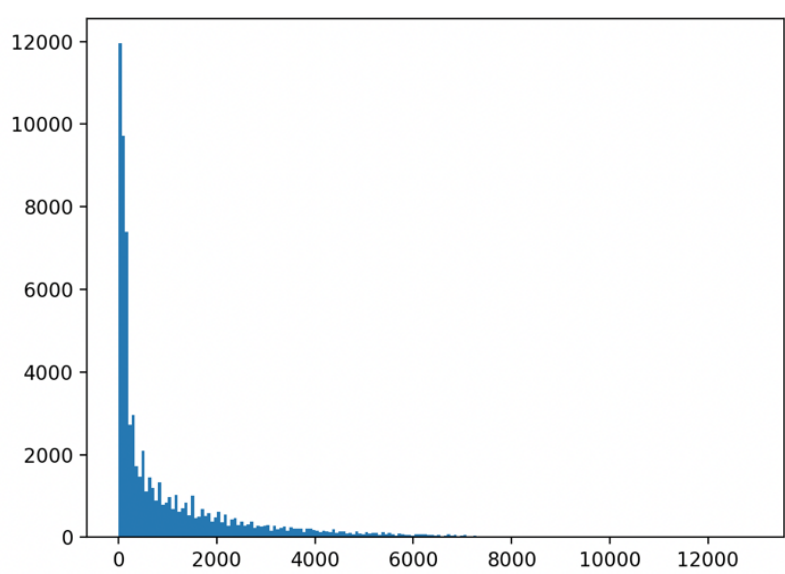

Fig. 4: Histogram of the throughput time [h]

the short, medium or large throughput time class.

TABLE II: Overview of multi-class target variable

\begin{tabular}{llll}
\hline & Short time & Medium time & Large time \\
\hline Delivery time & $0-6$ days & $7-39$ days & $>39$ days \\
\hline
\end{tabular}

\section{B. Evaluation of prediction}

We first perform a pretest with various machine learning algorithms in their standard parameter configuration [39]: Naïve Bayes, Neural Network, Support Vector Machine and Decision Tree. The results depicted in Table III are computed by performing a 70\%-30\% train-test-split on the first 2,000 data instances. We assume that those data instances all belong to the same concept as there is no significant change observable in the input data. Therefore, we can safely apply the machine learning algorithms without considering and handling concept drift. Note that prediction performance on later parts of the data set might be lower due to the challenges induced by concept drift.

TABLE III: Pretest with different models on subset of data

\begin{tabular}{l|c}
\hline Model & Accuracy \\
\hline Naïve Bayes & 0.767 \\
Neural Network & 0.805 \\
Support Vector Machine & 0.697 \\
ecision Tree & 0.740 \\
\hline
\end{tabular}

Naïve Bayes, Neural Networks and Decision Trees all achieve similar accuracy values. We choose Naïve Bayes classifier as the prediction algorithm which is due to two reasons: First, Naïve Bayes implements incremental learning which allows incremental uptates of the prediction model. Second, computational complexity of Naïve Bayes is rather low compared to other machine learning algorithms which allows frequent retraining of the model without the necessity for a large computational infrastructure.

Our work mainly focuses on the quantification and handling of concept drift. However, we do not have any knowledge 
whether there are any drifts at all in the data set or at which point in time they are occurring. Therefore, first of all, we analyze the impact of concept drift by applying a Naïve Bayes classification without any concept drift detection methodcalled "static model"- to the entire data set of 70,774 data instances. Subsequently, we apply Naïve Bayes classifier in combination with a Page-Hinkley test and ADWIN as drift detection methods. As evaluation metric, we use the accuracy by measuring how often the algorithm predicts the appropriate throughput time class. This metric is chosen since the instances are distributed equally over all three target classes.

The course of the accuracy of the static model without concept drift detection and incremental learning can be seen in Figure 5. The first 2,000 data instances are used for the initial training. Subsequently, we compute the first predictions and the accuracy level moves at around 0.7. Then, there is a first drop in accuracy after approximately 25,000 instances. However, the prediction performance recovers to around 0.7 shortly after. Subsequently, after approximately 35,000 instances, the prediction quality of the model decreases significantly. Supposedly, a concept drift has occurred because the model that is only trained on an initial data batch does not issue any useful prediction anymore. The accuracy over all predictions reaches 0.5400 .

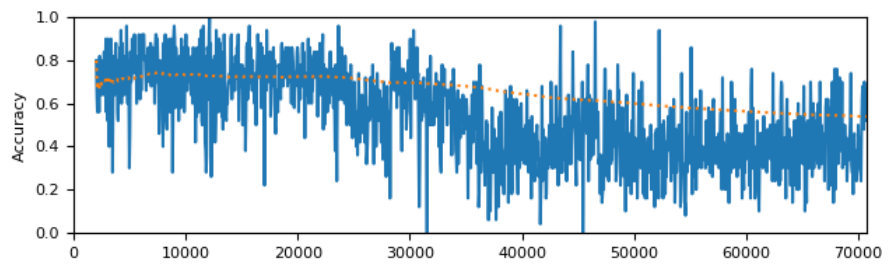

Fig. 5: Accuracy of Naïve Bayes without retraining and no drift detection method

As usual, it is difficult to determine the underlying reasons for this concept drift with certainty [36]. However, after a thorough analysis of additional data - which is not available the moment when the prediction is computed-we identify a possible explanation. The feature automation contains information about the percentage of process steps in the entire $\mathrm{P} 2 \mathrm{P}$ process which are executed automatically by corresponding information systems, while the other steps are executed manually. Thereby, the feature automation contains information about the level of automation in all processes. In order to analyze the development of this feature over time, we compute and plot a rolling mean (window $=1000$ ) of this feature which is depicted in Figure 6 .

At first, the rate of automation is rather stable before it rises abruptly and then fluctuates at a higher level. This plot clearly indicates on how the automation rate in the organization increases over time and thus, this may be one of the causes for concept drift and according changes in product delivery times. The sudden rise in automation maps rather well to the decrease in prediction accuracy in Figure 5. Relating to Section II, this abrupt change can be seen as a sudden concept drift. Since

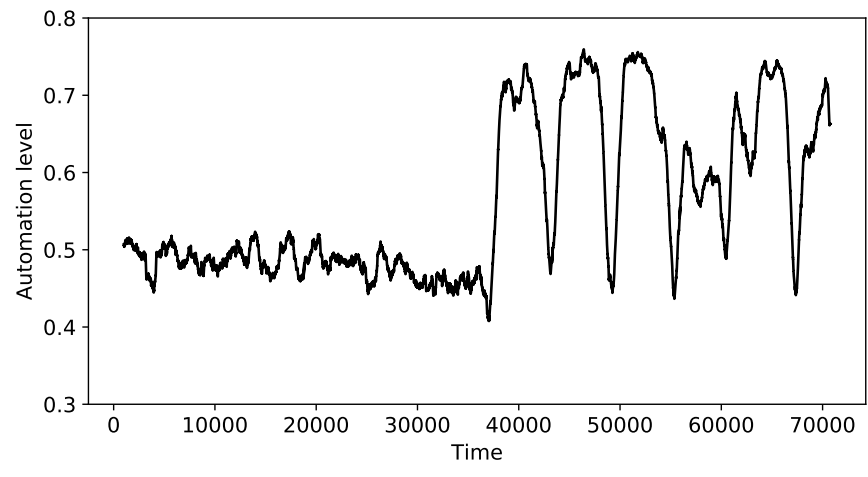

Fig. 6: Rolling mean (window 1000) of feature automation

this feature is not known at the time of prediction, it can be interpreted as a hidden context influencing the prediction.

Due to the detected drift, we apply a Page-Hinkley test as concept drift detection method in combination with the Naïve Bayes classifier. In case of drift, the model is retrained. The course of the accuracy of the model can be seen in Figure 7

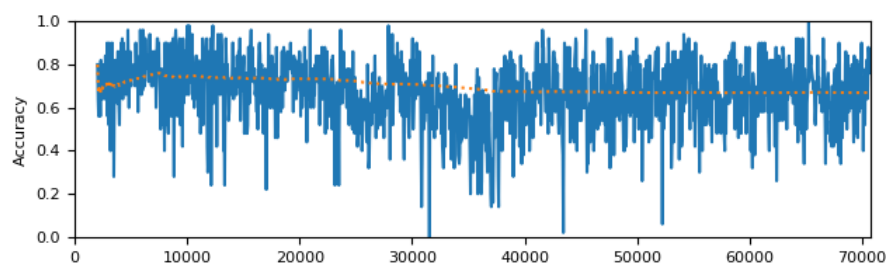

Fig. 7: Accuracy of NaïveBayes with Page-Hinkley

At the beginning, the figure looks similar to the model without drift detection (Figure 5 above). After approximately 35,000 instances, this model performs better because the drift is detected, and a retraining of the Naïve Bayes is triggered. The accuracy rises again and then stays same level with its corresponding fluctuations leading to an overall accuracy of 0.6732 (see Table IV). This is equivalent to a performance increase of $24 \%$. Furthermore, we extend this approach by activating incremental learning. This means that the model is constantly updated with new training data after it has issued prediction for those data. The application of incremental learning alone leads to a performance of 0.6717 . With both retraining and incremental learning, the overall prediction accuracy reaches 0.6938 .

TABLE IV: Performance of different data selection strategies on entire process mining data set

\begin{tabular}{c|c|c|c}
\hline Change detection & $\begin{array}{c}\text { Incremental } \\
\text { learning }\end{array}$ & Accuracy & $\begin{array}{c}\text { Performance } \\
\text { increase }\end{array}$ \\
\hline None (baseline) & No & 0.5400 & - \\
None & Yes & 0.6717 & $24.39 \%$ \\
Yes (Page-Hinkley) & No & 0.6732 & $24.67 \%$ \\
Yes (Page-Hinkley) & Yes & 0.6938 & $28.48 \%$ \\
\hline
\end{tabular}

We perform a grid search on the first 10,000 data instances in order to optimize the parameters of the drift detection 
method ADWIN $(\delta=0.001)$ and Page-Hinkley $(\lambda=0.6)$. With those parameters, we evaluate the different data selection strategies as discussed in Section III Table V depicts the accuracy score of a Naïve Bayes classifier with incremental learning in combination with a Page-Hinkley test or ADWIN as drift detection. Furthermore, we examine the influence of four different batch sizes $(500,1000,2000,5000)$ on the overall prediction accuracy. This refers to the amount of data instances which are provided to the model in case of retraining. The best results are marked in bold in Table V

As depicted in the table, the data selection strategy last performs always best. For our use case, Page-Hinkley appears to be the more suitable drift detector resulting in higher performance. Interestingly, the prediction accuracy decreases with increasing batch size which might indicate that the approach does not adapt fast enough with larger batches for retraining. Furthermore, the performance difference between the different data selection strategies also rises with the size of the batches. For instance, the difference between last and next for Page-Hinkley with batch size 500 equals 0.0073 in comparison to 0.0164 for Page-Hinkley with batch size 5000 .

TABLE V: Performance of different data selection strategies on process mining data set

\begin{tabular}{l|c|ccc}
\hline $\begin{array}{l}\text { Change detection } \\
\text { (batch size) }\end{array}$ & $\begin{array}{c}\text { Incremental } \\
\text { learning }\end{array}$ & Last & Mixed & Next \\
\hline Page-Hinkley (500) & Yes & $\mathbf{0 . 7 0 1 0}$ & 0.6961 & 0.6937 \\
Page-Hinkley (1000) & Yes & $\mathbf{0 . 6 9 6 5}$ & 0.6920 & 0.6903 \\
Page-Hinkley (2000) & Yes & $\mathbf{0 . 6 9 3 8}$ & 0.6845 & 0.6821 \\
Page-Hinkley (5000) & Yes & $\mathbf{0 . 6 8 4 2}$ & 0.6757 & 0.6678 \\
\hline ADWIN (500) & Yes & $\mathbf{0 . 6 8 5 6}$ & 0.6849 & 0.6843 \\
ADWIN (1000) & Yes & $\mathbf{0 . 6 8 5 4}$ & 0.6838 & 0.6825 \\
ADWIN (2000) & Yes & $\mathbf{0 . 6 8 0 3}$ & 0.6775 & 0.6750 \\
ADWIN (5000) & Yes & $\mathbf{0 . 6 7 5 8}$ & 0.6704 & 0.6675 \\
\hline
\end{tabular}

In general, the evaluation section clearly shows how the prediction performance can be increased by implementing drift handling strategies. Both, incremental learning as well as drift detection with retraining, have significant influence on the accuracy. Best results are achieved with the combination of both approaches.

\section{CONCLUSION}

Process mining relies more and more on techniques of machine learning. This work explores the challenge of concept drift for ongoing value creation in process mining. Specifically, we apply a concept drift detection algorithm on a use case which aims at predicting the delivery time for all purchase orders of a company. With this information, the company can optimize its internal service processes. We can show that concept drift handling significantly outperforms a static model in the given use case. Best results are achieved by combining incremental learning with retraining in case of concept drift. Regarding the best training data selection strategy for retraining, the last approach appears to be the best performing option. This means that data scientists should rely on the last collected data batch for the retraining of the prediction model.
The contribution of this paper is twofold. First, we systematically explain and depict the options for training data selection for the retraining of machine learning models in case of concept drift. Second, we apply and evaluate those options in a real-life use case in process mining where we can measure a significant increase in prediction performance from 0.5400 to 0.7010 . Regarding the managerial implication, this work clearly shows the importance of a continuous monitoring and adaptation scheme of predictive services in operation. Otherwise, they can quickly lose their validity and corresponding service offerings will not deliver expected benefits.

However, more research is required to understand the full effects of concept drift and the best strategies to deal with this problem. This work only describes and evaluates three options for the training data selection in case of retraining. Future work needs to evaluate more sophisticated approaches. Additional limitations regarding the use case arise through the transformation of the target variable from a regression problem into a multi-class classification problem. Furthermore, we only evaluate the data selection on one use case. More general recommendations could be derived by applying those options onto more use cases and benchmark data sets.

This paper clearly shows the importance of constant monitoring of predictive services for the detection of concept drifts. Frequent retraining and adaptations of a machine learning model are necessary requirements to keep and guarantee a high prediction performance. If practitioners consequently implement necessary monitoring activities, the economic benefits of predictive services and supervised machine learning solutions can still even be increased.

\section{REFERENCES}

[1] N. Kühl, M. Goutier, R. Hirt, and G. Satzger, "Machine learning in artificial intelligence: Towards a common understanding," in Proceedings of the 52nd Hawaii International Conference on System Sciences, 2019.

[2] M. I. Jordan and T. M. Mitchell, "Machine learning: Trends, perspectives, and prospects," Science, vol. 349, no. 6245, pp. 255--260, 2015.

[3] H. Chen, R. Chiang, and V. Storey, "Business Intelligence and Analytics: From Big Data to Big Impact," Mis Quarterly, vol. 36, no. 4, pp. 11651188, 2012

[4] R. Schüritz and G. Satzger, "Patterns of Data-Infused Business Model Innovation," in CBI 2016, 2016, pp. 133-142.

[5] T. H. Davenport, "Competing on analytics." Harvard business review, vol. 84 , no. 1 , pp. $98-107,134,2006$

[6] A. L. Ostrom, A. Parasuraman, D. E. Bowen, L. Patrício, and C. A. Voss, "Service Research Priorities in a Rapidly Changing Context," Journal of Service Research, 2015.

[7] P. G. J. Ching, "In AI we trust: Perceived value of advanced artificial intelligence in services," PhD thesis, The University of Queensland, 2018.

[8] L. Baier, N. Kühl, and G. Satzger, "How to Cope with Change? Preserving Validity of Predictive Services over Time," in Proceedings of the 52nd Hawaii International Conference on System Sciences (HICSS), 2019.

[9] L. Baier, F. Jöhren, and S. Seebacher, "Challenges in the deployment and operation of machine learning in practice," in Proceedings of the 27th European Conference on Information Systems (ECIS), 2019.

[10] A. Tsymbal, "The problem of concept drift: definitions and related work," Computer Science Department, Trinity College Dublin, vol. 4, no. C, pp. 2004-15, 2004.

[11] G. Widmer and M. Kubat, "Learning in the presence of concept drift and hidden contexts," Machine Learning, vol. 23, no. 1, pp. 69-101, 1996.

[12] J. Gama, I. Žliobaitè, A. Bifet, M. Pechenizkiy, and A. Bouchachia, "A survey on concept drift adaptation," ACM Computing Surveys, vol. 46, no. 4, pp. 1-37, 2014 
[13] W. M. P. van der Aalst, M. La Rosa, and F. M. Santoro, "Business Process Management," Business \& Information Systems Engineering, vol. 58, no. 1, pp. 1-6, feb 2016.

[14] M. Rosemann and J. vom Brocke, The Six Core Elements of Business Process Management. Berlin, Heidelberg: Springer Berlin Heidelberg, 2015, pp. 105-122.

[15] M. zur Muehlen and R. Shapiro, Business Process Analytics. Berlin, Heidelberg: Springer Berlin Heidelberg, 2015, pp. 243-263.

[16] W. Van Der Aalst, A. Adriansyah, A. K. A. De Medeiros, F. Arcieri and T. Baier, "Process mining manifesto," in Lecture Notes in Business Information Processing, 2012.

[17] C. C. Aggarwal, T. J. Watson, R. Ctr, J. Han, J. Wang, and P. S. Yu, "A Framework for Clustering Evolving Data Streams," Proceedings - 29th int. conf. on very large data bases, pp. 81-92, 2003.

[18] I. Zliobaite, "Learning Under Concept Drift: An Overview," arXiv preprint, 2010.

[19] G. I. Webb, R. Hyde, H. Cao, H. L. Nguyen, and F. Petitjean, "Characterizing concept drift," Data Mining and Knowledge Discovery, vol. 30, no. 4, pp. 964-994, 2016.

[20] E. S. Page, "Continuous inspection schemes," Biometrika, vol. 41, no. $1 / 2$, pp. $100-115,1954$

[21] A. Bifet and R. Gavalda, "Learning from time-changing data with adaptive windowing," in Proceedings of the 2007 SIAM international conference on data mining. SIAM, 2007, pp. 443-448.

[22] M. V. Manoj Kumar, L. Thomas, and B. Annappa, "Capturing the sudden concept drift in process mining," in CEUR Workshop Proceedings, 2015.

[23] W. M. P. Van der Aalst, Process mining: discovery, conformance and enhancement of business processes. Springer, 2011, vol. 2.

[24] W. M. Van der Aalst and A. J. Weijters, "Process mining: A research agenda," 2004.

[25] W. M. P. Van der Aalst, H. A. Reijers, A. J. M. M. Weijters, B. F. van Dongen, A. K. A. De Medeiros, M. Song, and H. M. W. Verbeek, "Business process mining: An industrial application," Information Systems, vol. 32, no. 5, pp. 713-732, 2007

[26] M. Dumas, M. La Rosa, J. Mendling, H. A. Reijers et al., Fundamentals of business process management. Springer, 2013, vol. 1

[27] W. M. Van der Aalst, M. H. Schonenberg, and M. Song, "Time prediction based on process mining," Information Systems, 2011.

[28] I. Verenich, M. Dumas, M. L. Rosa, F. M. Maggi, and I. Teinemaa, "Survey and cross-benchmark comparison of remaining time prediction methods in business process monitoring," ACM Transactions on Intelligent Systems and Technology (TIST), vol. 10, no. 4, pp. 1-34, 2019.

[29] A. E. Márquez-Chamorro, M. Resinas, and A. Ruiz-Cortes, "Predictive monitoring of business processes: a survey," IEEE Transactions on Services Computing, vol. 11, no. 6, pp. 962-977, 2017.

[30] C. Di Francescomarino, M. Dumas, F. M. Maggi, and I. Teinemaa, "Clustering-based predictive process monitoring," IEEE transactions on services computing, vol. 12, no. 6, pp. 896-909, 2017.

[31] R. P. J. C. Bose, W. M. P. Van der Aalst, I. Žliobaite, and M. Pechenizkiy, "Dealing with concept drifts in process mining," IEEE transactions on neural networks and learning systems, vol. 25, no. 1, pp. 154-171, 2013.

[32] A. Maaradji, M. Dumas, M. La Rosa, and A. Ostovar, "Detecting sudden and gradual drifts in business processes from execution traces," IEEE Transactions on Knowledge and Data Engineering, vol. 29, no. 10, pp. 2140-2154, 2017.

[33] A. Yeshchenko, C. Di Ciccio, J. Mendling, and A. Polyvyanyy, "Comprehensive process drift detection with visual analytics," in International Conference on Conceptual Modeling. Springer, 2019, pp. 119-135.

[34] A. Ostovar, S. J. Leemans, and M. L. Rosa, "Robust drift characterization from event streams of business processes," ACM Transactions on Knowledge Discovery from Data (TKDD), vol. 14, no. 3, pp. 1-57, 2020.

[35] B. Pfahringer, G. Holmes, and R. Kirkby, "New options for hoeffding trees," in Lecture Notes in Computer Science (including subseries Lecture Notes in Artificial Intelligence and Lecture Notes in Bioinformatics), 2007.

[36] I. Žliobaite, M. Pechenizkiy, and J. Gama, “An overview of concept drift applications," in Big data analysis: new algorithms for a new society. Springer, 2016, pp. 91-114.

[37] J. Montiel, J. Read, A. Bifet, and T. Abdessalem, "Scikit-multiflow: A Multi-output Streaming Framework," Journal of Machine Learning Research, 2018.

[38] G. E. P. Box and D. R. Cox, “An Analysis of Transformations,” Journal of the Royal Statistical Society: Series B (Methodological), 1964.
[39] F. Pedregosa, G. Varoquaux, A. Gramfort, V. Michel, B. Thirion, O. Grisel, M. Blondel, P. Prettenhofer, R. Weiss, V. Dubourg, J. Vanderplas, A. Passos, D. Cournapeau, M. Brucher, M. Perrot, and É. Duchesnay, "Scikit-learn: Machine learning in Python," Journal of Machine Learning Research, 2011 